Interview mit Norbert Thöne, Innenarchitekt

\title{
„In Praxisneubauten wird immer ein barrierefreies WC gefordert"
}

\section{Design, Funktionalität, Hygiene, Nachhaltigkeit: Worauf es bei der Planung des Patienten-WC wirklich ankommt, schildert Innenarchitekt Norbert Thöne aus seiner Berufspraxis.}

? Haben Sie für die Neugestaltung der Patiententoilette konkrete Empfehlungen parat? Oder zählt hier allein der Designwunsch des Arztes?

Dipl.-Ing. Norbert Thöne: Alle großen Sanitärhersteller bieten für den Gesundheitsbau spezielle sanitäre Einrichtungsgegenstände an, die sich aber oft am Bedarf in Krankenhäusern orientieren. Die normalen Patienten-WC-Anlagen können gerne designorientiert nach den Vorstellungen des Bauherrn ausgestattet werden.

? Welche Materialien setzen Sie in Sanitärräumen im Gesundheitsbau ein?

Thöne: Wichtig sind rutschhemmende Fußböden. Die Rutschhemmung wird in Klassen eingeteilt, in WC-Anlagen im öffentlichen Bereich muss der Belag der Klasse R10 entsprechen. Wir bevorzugen als Bodenfliesen Feinsteinzeug, das praktisch unverwüstlich ist, weil es keine glasierten Oberflächen hat. Feinsteinzeug hat eine sehr gute Abriebbeständigkeit und hohe Bruchsicherheit. Es ist in vielen attraktiven Designs erhältlich. Ebenfalls erhältlich sind Formteile für Sockel, sodass hier hygienisch einwandfreie Lösungen für die kritischen Übergänge zwischen Fußboden und Wandfliesen möglich sind.

? Stichwort Barrierefreiheit: Wie wird eine Toilette zum behindertengerechten WC?

Thöne: Es sind spezifische Einbauten erforderlich, wie unterfahrbare Handwaschbecken, auch Toiletten, die höher als üblich montiert werden und daher besser von Behinderten zu nutzen sind, zum Beispiel beim Umsetzen vom Rollstuhl aufs WC. Zudem ist ein Notruf vorzusehen, und die Tür zur Toiletten muss zugleich abschließbar und im Notfall von außen zu öffnen sein. Bei Neubauten werden von den Behörden grundsätzlich barrierefreie, behindertengerechte WC gefordert - eine Praxis ist ein öffentlich zugängliches Gebäude. Daher müssen die Vorgaben der DIN 18040-1 sowie 18024-2 erfüllt werden. Das berücksichtigen wir dann auch in der Planung.

? Längst nicht jede Patiententoilette hat ein Fenster. Welche Lüftungssysteme kommen infrage?

Thöne: WC-Anlagen werden meist über eine mechanische Abluftanlage entlüftet. Doch hierbei entsteht ein Unterdruck und man muss dafür sorgen, dass Zuluft nachströmen kann. Dies kann durch einen Raumluftverbund über Lüftungsrohre erfolgen, die einen anderen Raum mit natürlicher Lüftung mit dem WC verbinden. Oder über unterschnittene Türen - der Nachteil dieser Lösungen ist allerdings die Schallübertragung. Daher sollte mit einer Lüftungsanlage mit Zu- und Abluftleitungen geplant werden. Wenn diese zudem mit Wärmerückgewinnung arbeitet, ist sie auch unter energetischen Gesichtspunkten zu empfehlen.

? Geräusche sollen nicht nach außen dringen. Welche Lösungen bieten sich an, das Patienten-WC zu dämmen?

Thöne: Es gelten entsprechende DIN- und EU-Normen, die regeln, wie Leitungen und Zapfstellen auszuführen und zu dämmen sind. Sehr wichtig ist die Vermeidung von Schallbrücken - die unerwünschte Weiterleitung von Schall - bei der Installation. Idealerweise sollte man schon bei der Planung durch nebeneinanderliegende WC-Anlagen und gemeinsam genutzte Installationsschächte, die dann entsprechend gedämmt werden, Schwachstellen im Baubereich vermeiden. Wird der Bestand modernisiert, können Trockenbau- oder auch gemauerte Wände in der entsprechenden Dimensionierung sowie schalldämmende WC-Türen einen guten Schallschutz gewährleisten. Und der Einsatz einer Be- und Entlüftungsanlage vermeidet, wie schon erwähnt, ebenfalls die Übertragung des Schalls.

Das Interview führte Sabine Henßen.

\section{Intuitiv die Praxis steuern}

Bessere Visualisierung und Bedienbarkeit der Gebäudetechnik, das hat sich die Firma Jung aus Schalksmühle zum Ziel gesetzt. Ihr modulares Softwaresystem Facility-Pilot ist nun noch einfacher und komfortabler, weil intuitiv zu handhaben. Die Oberfläche des farbigen Touchscreens, dem Smart-Pilot, orientiert sich dabei logisch und nachvollziehbar an Funktionen, Räumen und selbst festgelegten Favoriten.

Die Smart-Pilots stehen in drei verschiedenen Größen zur Verfügung. Ihr elegantfuturistisches Design erinnert an das iPhone von Apple. Und für dieses steht auch die App "Smart Remote" zum Download im iTunes-Store bereit. „Smart Remote“ macht neben iPad und iPhone auch Smartphones mit Android-Betriebssystem zum Smart-Pilot für die Arztpraxis. Preis auf Anfrage. www.jung.de

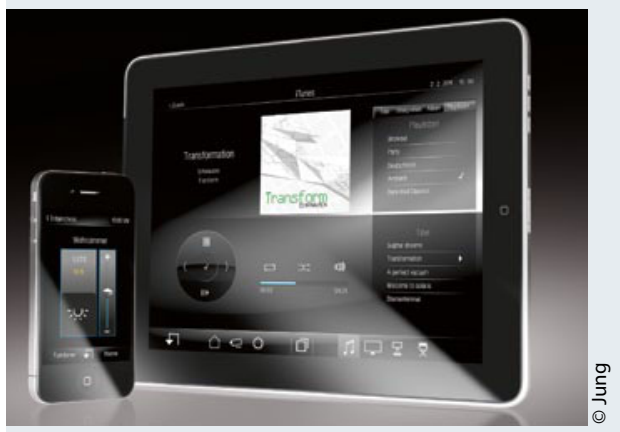

Natur-Design fürs Patienten-WC

Mit dem renommierten „red dot award“ für Produktdesign wurde die WaschtischSerie My Nature von Villeroy \& Boch ausgezeichnet. Das zentrale Gestaltungsmerkmal der Keramik - in runder oder ovaler Form erhältlich - ist die geschwungene Seitenlinie. Die geringe Stärke des Materials und die filigrane Formgebung unterstützen den Eindruck von Leichtigkeit. Neu ist das haptische, florale Dekor "Castanea", das für den Waschtisch zur Verfügung steht. Eine innovative Produktionstechnologie ermöglicht hierbei nicht nur sichtbare, sondern auch spürbare Dekore aufzubringen, die trotz der Tiefenstrukturen mühelos zu reinigen sind.

Die Befestigungslösung mit einer Konsole aus Kastanienholz erlaubt es, den Aufsatzwaschtisch auch als wandhängende Lösung einzusetzen. Preis: ab $300 €$. www.villeroy-boch.com 